\title{
Optical alignment of the JWST ISIM to the OTE simulator (OSIM): current concept and design studies \\ Bradley J. Frey ${ }^{*}$,, Pamela S. Davila ${ }^{a}$, John G Hagopian ${ }^{\mathrm{a}}$, James M. Marsh ${ }^{\mathrm{a}}$, Raymond G. Ohl ${ }^{\mathrm{a}}$, Joseph Sullivan ${ }^{\mathrm{b}}$, Geraldine A. Wright ${ }^{\mathrm{a}}$ \\ ${ }^{a}$ NASA Goddard Space Flight Center, Greenbelt, MD 20771 \\ ${ }^{\mathrm{b}}$ Ball Aerospace \& Technologies Corp., P.O. Box 1062, Boulder, CO 80306-1062
}

\begin{abstract}
The James Webb Space Telescope's (JWST) Integrated Science Instrument Module (ISIM) contains the observatory's four science instruments and their support subsystems. During alignment and test of the integrated ISIM at NASA's Goddard Space Flight Center (GSFC), the Optical telescope element SIMulator (OSIM) will be used to optically stimulate the science instruments to verify their operation and performance. In this paper we present the design of two cryogenic alignment fixtures that will be used to determine and verify the proper alignment of OSIM to ISIM during testing at GSFC. These fixtures, the Master Alignment Target Fixture (MATF) and the ISIM Alignment Target Fixture (IATF), will provide continuous, six degree of freedom feedback to OSIM during initial ambient alignment as well as during cryogenic vacuum testing. These fixtures will allow us to position the OSIM and maintain OSIM-ISIM alignment to better than 10 microns in translation and 250 micro-radians in rotation. We will provide a brief overview of the OSIM system and calibration and we will also discuss the relevance of these fixtures in the context of the overall ISIM alignment and verification plan.
\end{abstract}

Keywords: ISIM, OSIM, cryogenic, optical alignment, metrology

\section{INTRODUCTION}

The James Webb Space Telescope (JWST) is a space-based, infrared observatory currently being built by an international collaboration for NASA and will be launched in 2013. The JWST consists of three elements: the optical telescope element (OTE), the integrated science instrument module (ISIM), and the spacecraft element (sunshield and spacecraft bus). The OTE is $6.5 \mathrm{~m}$ in diameter and consists of 18 XXX segments of lightweighted beryllium XXX. Contained within the ISIM are four science instruments: the Near-InfraRed Camera (NIRCam), the Near-InfraRed multi-object Spectrograph (NIRSpec), the Mid-InfraRed Instrument (MIRI), and the Fine Guidance Sensor (FGS) with a near-infrared tunable filter camera (FGS-TF). Details about the design and science goals of the instruments is beyond the scope of this paper, but can be found elsewhere in the literature XXX add some references here...

The observatory verification is being done in an incremental fashion and at the top level is being done: by the science instruments by the SI vendors, at the integrated ISIM level with all the science instruments (at GSFC), and at the full observatory level (at Johnson Space Center). The ISIM structure and all the science instruments will be delivered to the GSFC ISIM team and will be integrated, aligned, and their performance verified as an integrated system during environmental testing in the Space Environment Simulator (SES) thermal vacuum chamber at GSFC. After the integrated ISIM element is aligned and verified at GSFC, it will be delivered to JSC where it will be integrated with the Optical Telescope Element (OTE) for end to end system level testing.

\footnotetext{
*Brad.Frey@nasa.gov, phone 1-301-286-7787, FAX 1-301-286-0204
} 


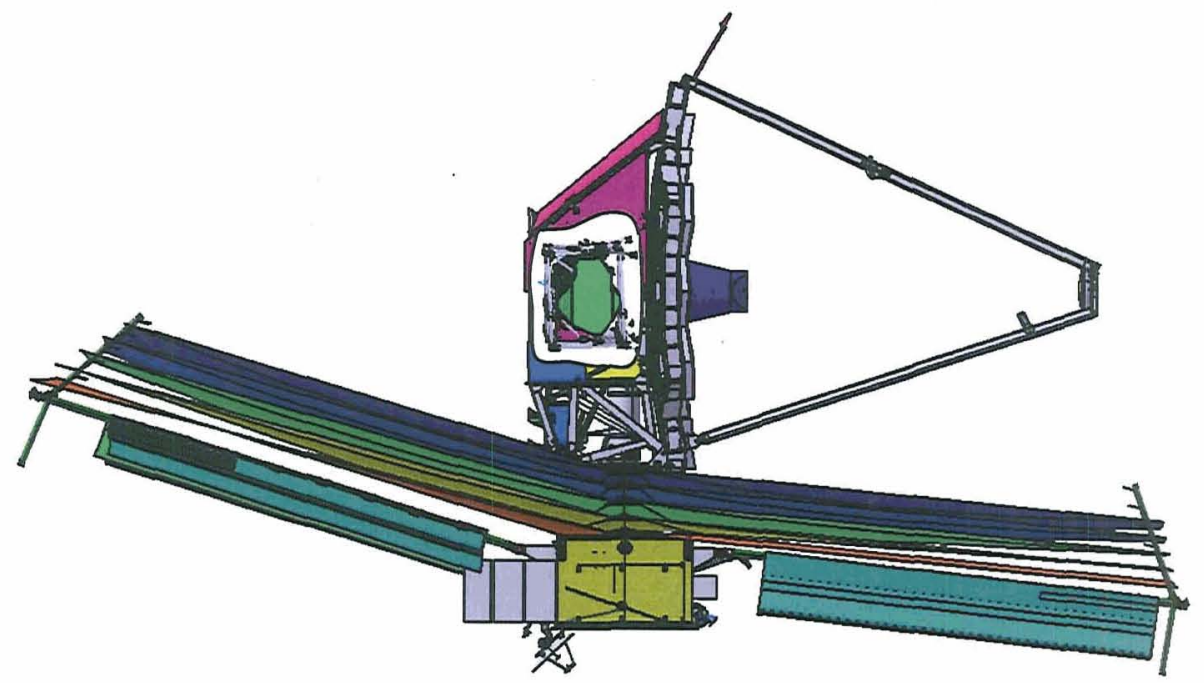

Figure 1: The James Webb Space Telescope

\subsection{TEST FLOW}

2. ISIM ALIGNMENT PLAN

3P

Once the ISIM structure is delivered to GSFC, it will undergo an extensive integration, alignment, and test plan both at ambient and operating environmental conditions. This testing will occur in the Space .... Facility (SSDIF) for ambient testing, and in the Space Environment Simulator (SES) for cryogenic vacuum testing. In addition to the optical alignment and testing, there will be a significant amount of additional testing by other disciplines (acoustics, vibration, etc.); those other portions of the test program will not be discussed in detail in this publication.

The first test of the ISIM structure will occur just prior to delivery of the structure to the GSFC ISIM integration and test (I\&T) team. This first test is the ISIM "cryo-set" test and currently consists of five thermal cycles of the bare, unpopulated ISIM structure (i.e. no science instruments) from ambient to operating temperature. Because of the nature of the composite material of which the ISIM is constructed, repeated thermal cycles are required to "set" the structure so that any internal stresses are relieved and its geometry is repeatable at operating temperature. During this cryo-set test, the photogrammetry (PG) metrology system XXX (reference Maria's paper here) will measure the relative locations of a series of metrology targets attached to the ISIM. The purpose of the cryo-set test is to characterize the repeatability of the locations of the PG targets at operating temperature to verify that the structure meets all appropriate requirements and performs as expected. Some of these metrology targets are located at the science instrument interface points (SIIPs); the PG system will verify that the locations of these interface points is within specification and the interface therefore is properly oriented and positioned to input no more than the nominal amount of strain into the mounts of the science instruments once they are installed on the ISIM. Finally, the PG system will characterize the relative locations of the ISIM, ICVF, IATF, and MATF. These measurements (and the repeatability of these measurements) will allow later measurements of the alignment state as measured by the IATF and MATF to be tied to the ISIM coordinate system. 
The next test of ISIM is "cryo-vac test \#1" and consists of the ISIM structure and ETU science instruments. (XXX do all the SIs have ETUs?) In addition, the OTE simulator (OSIM) will allow for optical alignment and testing of the populated ISIM structure. Since the ISIM is enclosed in a shroud cooled by liquid helium ( $\mathrm{LHe}$ ) to achieve the desired thermal environment for testing, and the OSIM is designed to operate outside the LHe shroud, but inside a liquid nitrogen shroud and operating at approximately $100 \mathrm{~K}$, the PG system cannot be used to metrologize the ISIM to OSIM alignment state. In order to properly align the ISIM - OSIM optical system, the two alignment fixtures - the IATF and MATF - must be used. The MATF is used as a surrogate for the nominal telescope coordinate system since the OSIM must be aligned such that its optical output is properly aligned to the nominal, on-orbit telescope output. This ensures that once the ISIM is aligned to the OSIM at GSFC, it will also be aligned to the telescope once the two are integrated at observatory level testing at the Johnson Space Center (JSC) later in the test program. Once the OSIM is properly aligned to the telescope coordinate system, the IATF is used to align the ISIM to the OSIM. The details of the alignment algorithms to be used are described in section XXX below. Not only are these two alignment fixtures critical to the initial alignment of the ISIM - OSIM system, but they will also be used to verify the alignment state throughout the extensive ISIM test program. In addition, the IATF will have to capability to monitor several aspects of the OSIM performance including wavefront quality and radiometric output simultaneous with science instrument testing. This OSIM monitor and calibration assembly (MCA) will be described in detail in section XXX. The test configuration for cryo-vac \#1 is shown in figure XXX. In this figure the science instruments and LHe shroud surrounding the ISIM have been removed for clarity so the nominal location of OSIM focal plane can be shown inside the ISIM.

The third ISIM test is the "cryo-vac test \#2" and consists of the ISIM structure populated with the flight science instruments. In addition, a flight-like thermal management system (TMS) will enclose the integrated ISIM structure to provide a well known thermal environment close to that expected on orbit. While the TMS will improve the thermal environment seen by the ISIM and provide a more flight-like test environment, it will preclude the use of the PG system which relies on a direct line of sight to the metrology targets on the ISIM structure. In place of using the PG system, the IATF and MATF will be the sole independent metrology means for aligning the ISIM - OSIM system and for verifying that alignment during SI testing. The repeatability of the measurements of the relative location and orientation of the IATF and MATF to the ISIM. coordinate system from the first two environmental tests (cryo-set and cryo-vac \#1) will be critical to the successful alignment of the system for this and the final cryo-vac test.

In cryo-vac \#2 several important measurements will be made. First, this test is the pre-acoustics and previbration test of the integrated ISIM structure. After cryo-vac \#2 is complete, the integrated ISIM with the science instruments (but not the IATF or MATF) will undergo the standard acoustics and vibration testing to demonstrate survival of simulated launch conditions. In addition, during cryo-vac $\# 2$ the global nominal focus (GNF) and global nominal pupil (GNP) positions will be measured, and the required ISIM kinematic mount (KM) strut length adjustment will be determined. The GNF and GNP measurements will be a determination of the "best-fit" location of the focal surface and pupil respectively as measured by the science instruments. In other words, each science instrument will measure the through focus images produced by the OSIM and finds the condition it deems to be the best focus and pupil location. This is repeated for all the science instruments, and the focal surface and pupil location that best satisfy all the instrument simultaneously are called the GNF and GNP respectively. This is then compared to the location of the telescope focal surface and pupil (as aligned by the MATF), and the difference (as measured by the IATF) is the KM required adjustment. Ideally, once the KMs are adjusted, the telescope focal surface and pupil will be coincident with the focal surface and pupil that provide the best overall science instrument performance on orbit. Both the IATF and MATF are critical to the proper alignment of the integrated ISIM to the nominal telescope optical output. 
The final ISIM test at GSFC is the "cryo-vac test \#3." During this test, the adjustments to the ISIM KMs that were made are verified and the final alignment of the ISIM to the nominal telescope output is checked. This test also verifies the performance of the science instruments after the vibration and acoustics testing and is the final confirmation of SI performance before integration with the OTE at JSC.

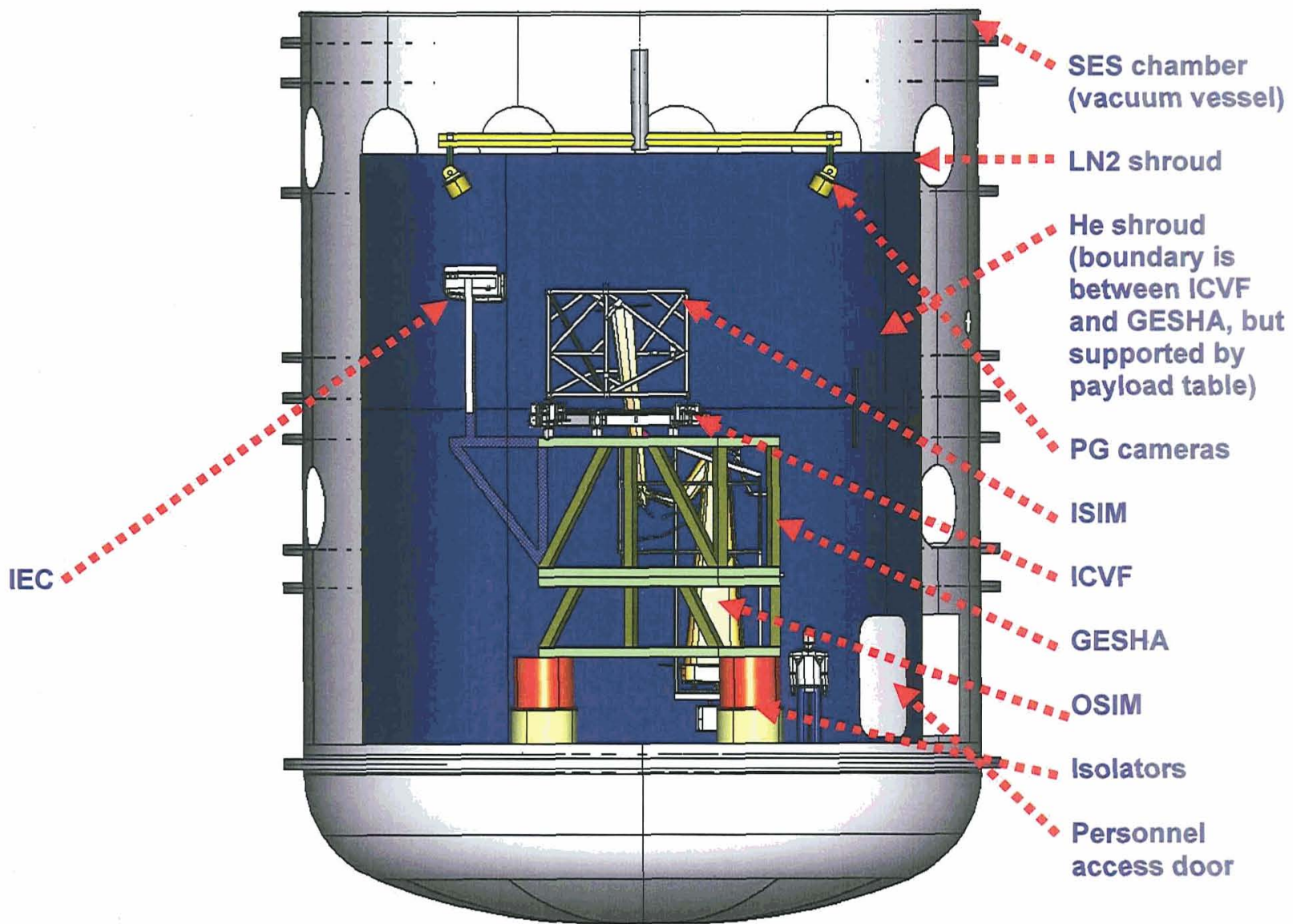

Figure XXX: Test configuration for cryo-vac test \#1 (with science instruments removed for clarity) in the SES chamber at GSFC.

\subsection{OSIM $\quad 1 \mathrm{p}$}

ISIM Element Cryogenic testing is performed in the Space Environment Simulator at the GSFC. The ISIM Element cryogenic testing verifies that the interfaces between ISIM and SI's are adequate to meet requirements at cryogenic operating temperature. The requirements that are verified include alignment, alignment stability and wavefront error. Photogrammetry is resident during the first cryogenic cycle of the ISIM element to directly measure the alignment changes of the SI benches in the ISIM coordinate system. The OTE Simulator (OSIM) is used for performance measurements of the SI's in the ISIM. Figure 13 shows the SES and ISIM and OSIM in the chamber. The OSIM is a deliverable to ISIM by Ball Aerospace Corporation. The OSIM design (see Figure 14) has evolved over time to match ISIM element verification requirements. OSIM operates at 100 Kelvins to allow testing of the Near and Mid Infrared Instruments without saturating the SI detectors with thermal radiation. The optical design of OSIM is quite simple, a spherical source plate contains several point sources for each instrument. This source plate resides behind a fold mirror with holes to allow the expanding sources to illuminate the primary mirror. The source platelfold mirror is positioned in front of the primary mirror center of curvature to create a focal surface that simulates that of the OTE. It was determined that the spherical aberration of the uncorrected OSIM design was 
preferable to the stringent alignment and fabrication requirements of a corrector plate. The OSIM design includes adjustable fold mirrors to allow beam steering and an accessible pupil to accommodate a number of stops.

The source module includes a variety of sources that operate at wavelengths from 0.6 to 5.0 microns. The source module resides outside of the SES to allow accessibility. Light is delivered to the source plate through fiber optics and light pipes. A minimum of five point sources are available to stimulate each SI. The sources must hove a capability for strobing at the 10-millisecond exposure level to allow vibration to be "frozen" out. Light is delivered from the source module to the source plate via fiber optics and light pipes. The fibers terminate at the surface of spherically shaped plate, which has decenter and despace adjustment capability. This allows the focal surface to be translated and refocused.

Referring to Figure 13, the ISIM and OSIM test configuration is shown. The ISIM is mounted above a Vibration Isolation System (VIS) within a cryogenic helium shroud. The OSIM is mounted onto the VIS as well to allow the ISIM and OSIM to move together during cooldown. A trade was performed to determine the best operational temperature for the OSIM based on manufacturing criteria, and thermal considerations. It was determined that 100 Kelvins allowed for ease of OSIM design and calibration, while enabling testing of the MIR channels of NIRCam and MIRI. The OSIM projects its stimuli through the opening where the Aft Optics System (AOS) will reside in the OTE to limit exposure of the hardware to the 100 Kelvins thermal radiation. The helium shroud encompasses ISIM and its support system, which are the ISIM Cryogenic Verification Fixture (ICVF) for the Engineering Unit ISIM tests and the Enhanced Pathfinder Backplane/BSF for the Flight Model ISIM. A helium shroud and a nitrogen shroud are below these support structures, with details of the interface to the VIS to be determined. A cryogenic shutter may be required between ISIM and OSIM to facilitate cooldown and limit radiative coupling between optical tests.

The JWST Integration and Test plan relies on a full verification of the ISIM element prior to delivery to Observatory testing. This serves two purposes, first it decouples the ISIM and OTE testing to allow maximum flexibility to isolate and remedy non-compliances for each element in parallel. Second, the OTE is significantly degraded in a 1-G environment, creating larger uncertainties in the performance parameters being verified. The OTE test configuration utilizes a sparse array of three autocollimating flats for verification further degrading the image quality. Therefore, the ISIM Element test with OSIM provides the best opportunity to verify the performance of everything other than the OTE.

\section{OSIM to ISIMAlignment}

The OSIM will project four out-of-field point sources to the simulated OTE focal surface. These point sources will be imaged on or near the LATF, which is installed on ISIM. Recall the IATF is fully characterized relative to the ISIM coordinate system and places position sensing photodiodes in a known position. Lateral effect cells are used to allow sufficient dynamic range to allow for OSIM and ISIM alignment uncertainties. The four lateral effect cells will report $x$-y positions of the reference point sources projected by OSIM. An algorithm will be employed to extract the six degree of freedom alignment error between OSIM and ISIM. A combination of adjustments on the OSIM fold mirrors, source plate and pupil mask will be commanded to align the OSIM to ISIM prior to optical testing.

Determination of Global Nominal Focus (GNF)

Once the OSIM is aligned to ISIM, the OSIM source plate is swept in focus to allow a series of through focus images to be collected for each scientific instrument. At least five point sources are activated for each scientific instrument to allow extraction of best focus and to determine if there is any focal plane tilt. $A$ variety of algorithms are under consideration for determining best focus6. The best OSIM focus position for each SI is determined, and a nominal offset calculated. All of the SI's except for MIRI are equipped with focus 
mechanisms that provide a minimum of $+3.1 \mathrm{~mm}$ of focus range. MIRI has a depth of field that allows it to meet performance requirements within $a+2.0 \mathrm{~mm}$ depth of field and slightly relaxed requirements over $a+$ $3.1 \mathrm{~mm}$ range. The best focus position for each SI that optimizes the entire suite of SI's will be determined from this set of measurements. All SI's should be within $1.13 \mathrm{~mm}$ of nominal focus position within the ISIM element. There is some additional offset allowed for grovity sag during ground testing. The set of SI focus positions is evaluated to provide a Global Nominal Focus position or GNF. There are actually four GNF positions, first there is a GNF for the OSIM testing with ISIM in the VI down orientation. The NIRCam, NIRSpec and FGS will be refocused to this position for further ISIM Element testing. Second, there is a calculated GNF position consistent with that expected for OTE and Observatory testing that offsets the SI focus position to compensate for SI gravity sag in the VI up orientation. Third, there is a nominal GNF position corresponding to the expected focus position for each SI onstation in zero gravity. Lastly, a new GNF position will be established when the Observatory is commissioned based on focus measurements obtained when the telescope is phased.

The OSIM will also contain an alignment diagnostics module (ADM) to facilitate OSIM system level alignment and OSIM - ISIM alignment. The ADM will contain an absolute distance meter - a laser based displacement measuring interferometer that has the capability to measure absolute distances - and an alignment telescope with a CCD detector and motorized focus mechanism. The ADM will be located behind the OSIM primary mirror and will view a series of targets that will be located at least on the primary mirror, OSIM source plate, pupil stop, the MATF, and possibly on the IATF. These targets will consist of a retroreflector for the absolute distance meter, a flat mirror with a pinhole and light source for the alignment telescope to measure tip, tilt, and decenter, and a Ronchi ruling that will be illuminated by a PIP generator and imaged by the alignment telescope to measure roll of the system about the master chief ray.

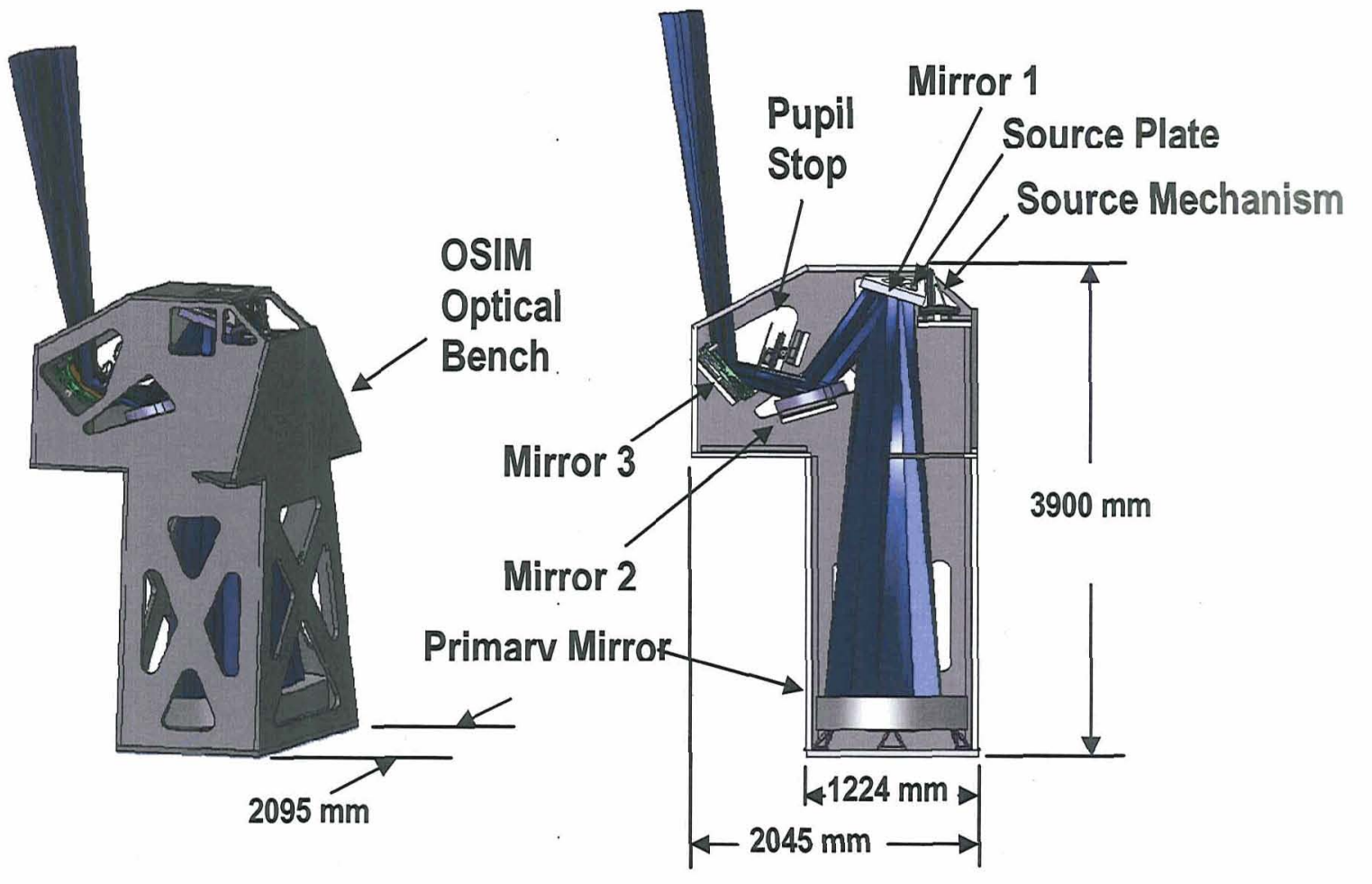

Figure XXX: The Optical Telescope Element Simulator (OSIM) 


\subsection{ISIM ALIGNMENT TARGET FIXTURE (IATF)}

The IATF is the alignment fixture that is used to align the ISIM to the OSIM as described in Section XXX. It will have a direct mechanical interface with the ISIM and because it is ground support equipment that will be not be used on orbit, it must meet requirements to ensure its presence does not alter the performance of ISIM or the SIs in any way. In other words, if the alignment state, thermal environment, structural repeatability on cool-down, or any other optical, mechanical, or thermal measure of performance changes after the IATF is removed, the alignment done at GSFC will not be a valid prediction of on-orbit performance. These constraints along with the stringent alignment measurement requirements have driven the design of the IATF and its modes of operation (see figure XXX).

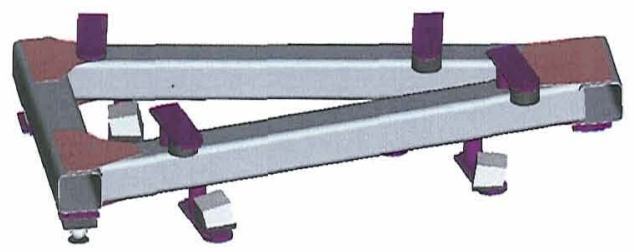

Figure XXX: Current IATF design concept. The overall dimensions of the IATF are approximately $1 \mathrm{~m} \times 0.5 \mathrm{~m} \times 150$ $\mathrm{mm}$ with the main structure constructed of $75 \mathrm{~mm}$ square composite tubes.

The mode of operation of the IATF is as follows: The IATF will use four array type detectors to measure the centroids of four field points projected by the OSIM onto the focal plane. The four field points will be measured only by the IATF and are independent of the field points generated by the OSIM to stimulate the science instruments. The relative locations of these four detectors to each other and to the photogrammetry targets will be carefully measured using a coordinate measuring machine to calibrate their locations to better than XXX microns. Once the centroids of the four OSIM source points have been measured, their absolute locations can be translated into absolute alignment information using the IATF calibration results and the photogrammetry results relating the location of the IATF to the ISIM coordinate system. Using an optical model of the OSIM-IATF system, we have determined the expected apparent centroid shift relative to the nominal focal plane as a function of IATF motion; these results are described in Table XXX. These results allow us to generate an overall error budget to describe how well we must measure the source point centroids taking into account other sources of error including: detector centroid accuracy, knowledge of relative detector locations, knowledge of relative locations of detectors to PG targets, and accuracy of PG IATF-ISIM coordinate system measurements. Since this error budget is divided between several quantities that must then be combined to meet the requirements listed in Table XXX, the contribution of any of the individual sources of error must be small. To date, the most challenging of these requirements to meet has been the knowledge of the relative positions of the detectors to each other - currently the allocation for this portion of the error budget is 6 microns - at cryogenic temperatures. At the time of publication, we are in the process of modeling the expected alignment stability and repeatability of the IATF system as a function of operating temperature.

Table XXX: IATF alignment requirements and alignment technique sensitivity

\begin{tabular}{|c|c|c|c|}
\hline Degree of freedom & $\begin{array}{c}\text { Alignment knowledge } \\
\text { requirement }\end{array}$ & $\begin{array}{c}\text { Sensitivity (centroid } \\
\text { motion at the focal } \\
\text { plane/misalignment) }\end{array}$ & $\begin{array}{c}\text { Required centroid } \\
\text { knowledge (incl. all } \\
\text { error sources) }\end{array}$ \\
\hline $\mathrm{dV1}$ (focus) & $37 \mathrm{microns}$ & $271 \mathrm{microns} / \mathrm{mm}$ & 10 microns \\
\hline $\mathrm{dV} 2$ & $4 \mathrm{microns}$ & $1 \mathrm{~mm} / \mathrm{mm}$ & 4 microns \\
\hline $\mathrm{dV} 3$ & $7 \mathrm{microns}$ & $1 \mathrm{~mm} / \mathrm{mm}$ & $7 \mathrm{microns}$ \\
\hline$\Theta \mathrm{V} 1$ & $0.02 \mathrm{mrad}$ & $389 \mathrm{microns} / \mathrm{mrad}$ & $8 \mathrm{microns}$ \\
\hline$\Theta \mathrm{V} 2$ & $0.22 \mathrm{mrad}$ & $72 \mathrm{microns} / \mathrm{mrad}$ & $16 \mathrm{microns}$ \\
\hline$\Theta \mathrm{V} 3$ & $0.17 \mathrm{mrad}$ & $68 \mathrm{microns} / \mathrm{mrad}$ & $12 \mathrm{microns}$ \\
\hline
\end{tabular}


In addition to the tight alignment and calibration requirements on the IATF, we also have the requirement to operate over the entire temperature range of ISIM testing: $313 \mathrm{~K}$ to $30 \mathrm{~K}$. This poses several interesting challenges, one of which is choosing a detector that will meet all of these requirements simultaneously. We are currently considering two detector options: an off the shelf CCD camera and large format $\mathrm{HgCdTe}$ arrays similar to those used in NIRCam and NIRSpec, though not necessarily of science grade quality. Each of these options poses some technical challenges and solutions for those challenges are being investigated at the time of publication.

The commercially available CCD camera is an attractive option due to the small pixel size ( $\sim 5-7$ microns), small package, ease of operation (plug and play firewire interface), and low price. The drawbacks to the CCD camera option include large power dissipation $(2 \mathrm{~W})$ and operation at $30 \mathrm{~K}$. We have done some preliminary testing that shows the CCD cameras will work in a $30 \mathrm{~K}$ environment, if thermally isolated and allowed to "self-heat" themselves to around $80 \mathrm{~K}$ or higher. Unfortunately this amount of power dissipation is unacceptable for during ISIM testing due to the close vicinity to the science instruments and their sensitivity to thermal gradients. We are currently investigating options for better isolating the CCDs and removing any power dissipated by them from the ISIM thermal environment.

The $\mathrm{HgCdTe}$ arrays are an attractive option from the thermal aspect due to their natural operation at $35 \mathrm{~K}$ and low power dissipation $(<20 \mathrm{~mW})$ during operation. The large pixel size of 18 microns could make centroid measurement to the required levels challenging, though not insurmountable. Unfortunately room temperature operation of such an array is not practical due to the extremely high dark current inherent to these detectors while operating at higher temperatures.

In addition to the requirements to measure and verify ISIM - OSIM alignment, the IATF will also be used to measure several aspects of OSIM performance in real time during ISIM testing. This monitor and calibration assembly (MCA) will include a point diffraction interferometer (PDI) with pupil imaging capabilities to measure the wavefront quality of the OSIM and two radiometer modules to measure the absolute radiometric output of the OSIM (the MCA is not shown in figure XXX). The MCA has been designed such that pinhole of the PDI is located on the OSIM focal surface 18 inches below the IATF into the ISIM structure. The camera for the PDI will be located near the IATF structure to minimize the potential thermal load on the SIs. The two radiometer modules (one is required, the second is for redundancy) are attached on the sides of the PDI and are located just above the OSIM focal plane to provide a XXX $\mathrm{mm}$ spot size on the $7 \mathrm{~mm} \mathrm{InSb}$ single element radiometer. These radiometers are NIST calibrated and will provide absolute radiometry accurate to $20 \%$ for NIRCam.

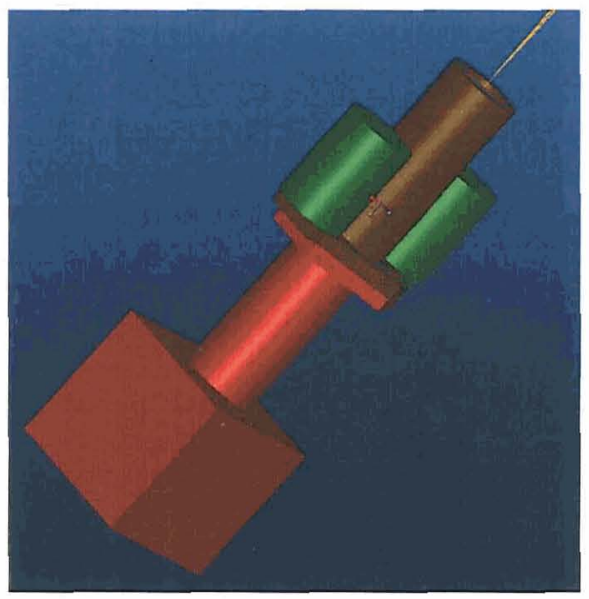

Figure XXX: OSIM monitor and calibration assembly

\subsection{IATF BREADBOARD TESTING}

One additional challenge that must be overcome is to develop the algorithms to prove that once we have measured the centroids of the four OSIM source points we can extract the needed 6 degree of freedom OSIM - ISIM alignment 
information. Some work has been done in this area though optical models, but we have also constructed a breadboard of the IATF structure to prove that the technique will work with real, laboratory data (see Figure XXX). Using almost entirely off the shelf parts we have constructed a full scale model of the IATF structure with 4 commercially available CCD cameras, and simulated the optical output of OSIM by aligning 4 "point sources" (pinholes illuminated by LEDs) at the appropriate location relative to the IATF to represent the OSIM focal plane. Off the shelf singlet lenses were used to reimage the sources onto the CCDs at a magnification of 0.25 . Using a nominal design model as calibration for the algorithms, our preliminary results show that we can indeed extract the necessary alignment information from the centroid data. Detailed sensitivity testing is ongoing at the time of publication.

\section{Insert photo of lab setup here}

\subsection{MASTer AlignMent TARget Fixture (MATF)}

The current baseline for the alignment technique on the MATF is a series of passive alignment targets that can be seem by the OSIM alignment diagnostics module. A technique similar to that of the IATF is not practical for the MATF due to its location relative to the OSIM pupil. At the current MATF location, almost all of the current OSIM source points overlap, making an imaging technique with the MATF impossible to do simultaneous with SI testing. Even without simultaneous SI testing, the MATF imaging system would have to be removed after alignment to prevent vignetting of the SI fields of view, requiring an additional cryogenic mechanism to be added to the system. Instead, we have opted for a passive alignment system that - although we still can't measure our alignment simultaneous with SI testing - will minimize our thermal impact and significantly simplify our mechanical design. The capabilities of the OSIM alignment diagnostics system include an absolute distance meter (laser based displacement measuring interferometer system) and alignment telescope with CCD camera and remotely controllable focus adjustment mechanism.

\section{CONCLUSION $1 / 2 p$}

Alignment of the integrated JWST ISIM structure including its four science instruments at cryogenic temperatures is a challenging task. Using the OTE simulator (OSIM) and two alignment fixtures - the ISIM alignment target fixture and the master alignment target fixture - we have outlined a plan by which the ISIM and SIs can be properly aligned to the 
optical output of the OSIM and to the appropriate observatory coordinate system. We have generated a preliminary design of this ground support equipment and have started to model its optical performance to show that it will meet all alignment requirements. We have also constructed a laboratory testbed to prove our alignment technique will work and to investigate the performance of our alignment algorithms.

\section{ACKNOWLEDGEMENTS}

The authors would like to thank...

\section{REFERENCES}

Hagopian's ISIM alignment paper

Nowak PG paper 